\title{
Characterization of Conventional One-Step Sodium Thiosulfate Facilitated Gold Nanoparticle Synthesis
}

\author{
Scott-Eugene Saverot ${ }^{1 \dagger}$, Laura M Reese ${ }^{2,3 \dagger}$, Daniela Cimini ${ }^{4}$, Peter J Vikesland ${ }^{3,5}$ and Lissett Ramirez Bickford ${ }^{2,3,6^{*}}$
}

\begin{abstract}
Gold-gold sulfide nanoparticles are of interest for drug delivery, biomedical imaging, and photothermal therapy applications due to a facile synthesis method resulting in small particles with high near-infrared (NIR) absorption efficiency. Previous studies suggest that the NIR sensitivity of these nanoparticles was due to hexagonally shaped metal-coated dielectric nanoparticles that consist of a gold sulfide core and gold shell. Here, we illustrate that the conventional synthesis procedure results in the formation of polydisperse samples of icosahedral gold particles, gold nanoplates, and small gold spheres. Importantly, through compositional analysis, via UV/vis absorption spectrophotometry, transmission electron microscopy (TEM), and energy dispersive $x$-ray spectroscopy (EDS), we show that all of the nanoparticles exhibit identical face center cubic (FCC) gold crystalline structures, thus suggesting that sulfide is not present in the final fabricated nanoparticles. We show that icosahedrally shaped nanoparticles result in a blue-shifted absorbance, with a peak in the visible range. Alternatively, the nanoplate nanoparticles result in the characteristic NIR absorbance peak. Thus, we report that the NIR-contributing species in conventional gold-gold sulfide formulations are nanoplates that are comprised entirely of gold. Furthermore, polydisperse gold nanoparticle samples produced by the traditional one-step reduction of HAuCl4 by sodium thiosulfate show increased in vitro toxicity, compared to isolated and more homogeneous constituent samples. This result exemplifies the importance of developing monodisperse nanoparticle formulations that are well characterized in order to expedite the development of clinically beneficial nanomaterials.
\end{abstract}

Keywords: Gold-gold sulfide; Nanoplates; Photothermal therapy; Theranostic nanoparticles

\section{Background}

Gold-based nanoparticles have received immense research attention due to their unique optical properties, biocompatibility, and ease of surface modification [1]. Careful manipulation of the resonance oscillation of conductive electrons, or surface plasmon resonance (SPR), enables these particles to be utilized in an array of biomedical applications including photothermal therapy and imaging [2]. Gold nanocages, nanorods, nanoshells, and nanoplates have all been investigated due to the location of their SPR in the near-infrared (NIR) region, where tissue attenuation

\footnotetext{
* Correspondence: bickford@vt.edu

${ }^{\dagger}$ Equal contributors

${ }^{2}$ Department of Biomedical Engineering and Mechanics, Virginia Tech, 325 Stanger Street, 310 Kelly Hall (MC 0298), Blacksburg, VA 24061, USA

${ }^{3}$ Virginia Tech Center for Sustainable Nanotechnology, Virginia Tech, Kelly

Hall, Blacksburg, VA 24061, USA

Full list of author information is available at the end of the article
}

is minimized and light penetration is maximized [3]. One specific formulation of nanoparticles of particular interest is gold-gold sulfide nanoparticles (GGS NP). To date, GGS NP have been investigated in pre-clinical studies spanning chemotherapy delivery to tumors [4] to immunotargeted cancer therapy and imaging [5-8]. GGS NP are reportedly advantageous over other gold formulations due to their rapid and facile one-step synthesis, superior NIR absorption efficiency (98\%) and optimal size for accumulation within tumors in vivo [5]. In spite of their demonstrated potential and compelling characteristics, GGS NP have not reached the level of human clinical testing seen with other gold moieties, such as gold nanoshells (NCT00848042, NCT016794) or gold colloid (NCT0035690). Initially, GGS NP were proposed as having an icosahedral, core-shell nanostructure $[9,10]$. Subsequent studies have disputed this, asserting that the 
particles are actually gold-aggregates or pure gold nanoparticles $[11-16,6]$. Based on the original published synthesis procedures, GGS NPs can be characterized as having two SPR bands: a $520 \mathrm{~nm}$ peak attributed to small spherical gold nanoparticles and an $800 \mathrm{~nm}$ peak contributed by a heterogeneous combination of predominately icosahedra, nanoplates, and irregularly shaped asymmetric nanoparticles [6]. To realistically receive approval from the Food and Drug Administration (FDA) for ultimate in vivo applications, nanoparticle solutions must be homogenous and well characterized to ensure consistent particle performance [17]. This would facilitate accurate prediction of the biodistribution and ultimate fate of the nanoparticles, which is necessary for identifying effectiveness as well as potential health hazards and long-term toxicity. In an effort to maximize monodispersity, and thus increasing their clinical potential, recent alternative synthesis methods have been developed including: synthesis of $\mathrm{Au}_{2} \mathrm{~S}$ cores from $\mathrm{H}_{2} \mathrm{~S}$ gas and potassium dicyanoaurate followed by pure gold shell growth, synthesis with an alternate gold precursor and radiation-induced reduction, and a two-step synthesis method that resulted in increased nanoplate formation [18-20]. Additional efforts have focused on eliminating small spherical gold impurities (with an SPR absorbance at $~ 520 \mathrm{~nm}$ ) using physical methods such as filtration and dialysis [21, 22]. While these distinct fabrication procedures are in various stages of research and development with their own advantages, we proceed with a detailed analysis of conventional GGS NP fabrication to clarify unknown issues for potential future nanomedicine applications, namely, a thorough characterization and elucidation of the structure of these nanoparticles and their elemental composition, confirmation of the nanoparticulate species that predominately contributes to the NIR peak, and a closer examination of sample polydispersity and cellular toxicity. This will lead to future studies aimed at creating optimal monodisperse solutions in order to minimize undesirable toxicity $[17,23]$.

\section{Methods}

\section{Gold-Gold Sulfide Nanoparticle Synthesis}

Nanoparticles were synthesized based on previously described methods using conventional fabrication procedures [5, 7]. Briefly, $2 \mathrm{mM}$ hydrogen tetrachloroaurate (III) trihydrate $\left(\mathrm{HAuCl}_{4}: 3 \mathrm{H}_{2} \mathrm{O}\right.$, Sigma Aldrich) and $1 \mathrm{mM}$ sodium thiosulfate $\left(\mathrm{Na}_{2} \mathrm{~S}_{2} \mathrm{O}_{3}\right.$, Sigma Aldrich) were prepared in Milli-Q water in amber bottles and allowed to age for 3 days prior to synthesis. $\mathrm{Na}_{2} \mathrm{~S}_{2} \mathrm{O}_{3}$ was added to the $\mathrm{HAuCl}_{4}$ in a round bottom flask under mild stirring at room temperature $\left(25^{\circ} \mathrm{C}\right)$ and allowed to react for $1 \mathrm{~h}$ at a volumetric ratio of 1.03:1, forming the GGS NP. For temperature studies, the nanoparticles were similarly prepared but stirring occurred on either a hot plate $\left(50{ }^{\circ} \mathrm{C}\right)$, under refrigeration $\left(4{ }^{\circ} \mathrm{C}\right)$, or on ice $\left(0^{\circ} \mathrm{C}\right)$. All nanoparticle samples were centrifuged twice at $3200 \mathrm{~g}$ for $40 \mathrm{~min}$, and pellets were resuspended in $\mathrm{H}_{2} \mathrm{O}$ to a final optical density (OD) of 1.3. Particles were PEGylated by mixing $1 \mathrm{~mL}$ of 250 mM PEG-SH (Laysan Bio $2000 \mathrm{MW}$ ) in ultrapure water with $9 \mathrm{~mL}$ of the nanoparticle suspension on ice, followed by constant agitation overnight at $4{ }^{\circ} \mathrm{C}$ (Rotoflex). PEGylated particles were centrifuged at $3200 \mathrm{~g}$ for $40 \mathrm{~min}$ at $10{ }^{\circ} \mathrm{C}$ to remove excess PEG and were stored in the refrigerator at $4{ }^{\circ} \mathrm{C}$ until further use.

\section{Nanoparticle Characterization}

$\mathrm{UV} /$ vis spectrophotometry analysis was performed using a Cary 60 UV/vis spectrophotometer (Agilent). For transmission electron microscopy (TEM) analysis, $5 \mu \mathrm{L}$ of nanoparticle solution $(1 \mathrm{mg} / \mathrm{mL})$ were drop cast onto a carbon 200-mesh copper grid (Ted Pella) and the grids were covered and air-dried overnight, prior to imaging. TEM imaging was performed with a JEOL 2100 field thermionic emission TEM equipped with a silicon-drifted detector-based energy dispersive $x$-ray spectroscopy (EDS) system. ImageJ software was utilized to determine nanoparticle yields. Selected area electron diffraction (SAED) patterns were obtained using the field-limiting aperture, and fast Fourier transforms (FFT) were obtained in high-resolution mode. Determination of the 3D structure of the nanoparticles was obtained using tilt tomography and a tilt angle of $30^{\circ}$. DigitalMicrograph (Gatan) was used to analyze diffraction patterns, for FFT, to measure d-spacing, and to determine particle sizes.

\section{Growth Kinetics}

To evaluate the nanoparticle growth kinetics using conventional formulations, nanoparticles were synthesized in a microwave to accelerate nucleation. $\mathrm{HAuCl}_{4}$ and $\mathrm{Na}_{2} \mathrm{~S}_{2} \mathrm{O}_{3}$ were mixed and microwaved for $10 \mathrm{~s}$ at $450 \mathrm{~W}$ power to induce nuclei formation and then immediately quenched on ice to prevent further nanoparticle growth. As a control, another subset of nanoparticles was microwaved under identical conditions and allowed to react at room temperature for $1 \mathrm{~h}$.

\section{Seed-Mediated Growth}

A commonly used approach for nanoparticle synthesis that isolates nucleation from growth is a seed-mediated method. Small monodisperse nuclei are formed and then allowed to react with additional metal salt and a reducing agent to form larger particles of consistent sizes. Gold seeds were formed based upon methods previously described by Haiss et al. [24]. Briefly, $1 \mathrm{~mL}$ of $1 \mathrm{wt} \%$ $\mathrm{HAuCl}_{4}$ was first added to $90 \mathrm{~mL}$ Milli-Q $\mathrm{H}_{2} \mathrm{O}$, followed by the addition of $2 \mathrm{~mL}$ of $38.8 \mathrm{mM}$ sodium citrate while the solution stirred for $2 \mathrm{~min}$. Fresh 0.075 wt $\% \mathrm{NaBH}_{4}$ was then added drop wise until the solution turned a red wine color (indicative of seed development), followed by 
stirring for five additional minutes. Next, $2 \mathrm{~mL}$ of $1 \mathrm{mM}$ $\mathrm{Na}_{2} \mathrm{~S}_{2} \mathrm{O}_{3}$ were mixed with $0.25 \mathrm{~mL}$ of gold seed and aged for $24 \mathrm{~h} .1 .75 \mathrm{~mL}$ of $2 \mathrm{mM} \mathrm{HAuCl}_{4}$ was added to the solution of gold seed and $\mathrm{Na}_{2} \mathrm{~S}_{2} \mathrm{O}_{3}$ while stirring and allowed to react for $1 \mathrm{~h}$ at room temperature.

\section{Size-Selective Separation of Polydisperse Nanoparticles}

Two different methods for nanoparticle size separation were used: density gradient separation and microfiltration. Density gradient separation was based upon previous methods, wherein $2 \mathrm{~mL}$ gradient glycerol concentrations (ranging from $30-90 \%$ of glycerol by mass) were first layered in a $15-\mathrm{mL}$ conical tube [25]. Next, $0.8 \mathrm{~mL}$ of bare particles were aliquoted on the topmost layer, and the samples were centrifuged for $40 \mathrm{~min}$ at $3200 \mathrm{~g}$ at $40{ }^{\circ} \mathrm{C}$. Distinct bands of nanoparticles were extracted with a dispensing needle, centrifuged at $4722 \mathrm{~g}$ for $8 \mathrm{~min}$ to remove the glycerol, and resuspended in water. For microfiltration, PEGylated nanoparticles were sequentially passed through $0.22-, 0.1-$, and $0.05-\mu \mathrm{m}$ filters (Polycarbonate, Sterlitech). The 0.22- and 0.1- $\mu \mathrm{m}$ filters (PVDF Millex) were sonicated in a water-filled beaker for $30 \mathrm{~min}$ to remove the captured nanoparticles. Nanoplate concentration in different samples was determined by physically counting the number of nanoplates out of 100 particles in the field of view via TEM imaging.

\section{Nanoparticle Stability}

Prior to performing toxicity studies, PEGylated nanoparticles were assessed for stability. Nanoparticles were suspended in DMEM-F12 media at an OD of 0.66. Stability was monitored every $30 \mathrm{~min}$ over $24 \mathrm{~h}$ using the scanning kinetics function of the UV/vis spectrophotometer. The absorbance at the NIR resonant peak was normalized over time. A significant decrease in absorbance reflects nanoparticle instability due to protein absorption and the settling of the particles [26].

\section{Nanotoxicity Studies}

To evaluate potential undesirable toxic effects of the nanoparticles towards healthy cells, PEGylated nanoparticles were incubated with hTERT-RPE-1 (RPE-1) cells, an immortalized human telomerase reverse transcriptaseretinal epithelial cell line. GGS NP have formerly undergone testing for photothermal therapy of solid tumors, which develop within epithelial tissues. RPE-1 cells represent an ideal model for toxicity tests, given that epithelial cells are the most abundant healthy cell type found in the immediate proximity of tumors. Furthermore, these cells do not undergo senescence, allowing longer experiments to be conducted as compared to other commonly used cell types [27]. Toxicity assessment was based on previously established methods [7, 28]. Briefly, RPE-1 cells were plated in 96-well plates at a density of 100 cells $/ \mathrm{mm}^{2}$ and allowed to adhere for $24 \mathrm{~h}$ at $37{ }^{\circ} \mathrm{C}, 5 \% \mathrm{CO}_{2}$. Cells were incubated with media containing the PEGylated nanoparticles at concentrations ranging from 5-100 $\mu \mathrm{g} /$ $\mathrm{mL}$. These concentrations represent the maximum anticipated amount of nanoparticle exposure to normal cells based on tumor accumulation due to systemic and local injections, as previously reported [28]. Nanoparticles were incubated with cells for $24 \mathrm{~h}$ at $37^{\circ} \mathrm{C}, 5 \% \mathrm{CO}_{2}$. Cell toxicity was analyzed using both the Celltiter-glo ${ }^{\circ}$ cell viability assay (Promega) and the lactase dehydrogenase (LDH) cytotoxicity assay (Piercenet). Celltiter-glo quantifies the concentration of adenosine triphosphate (ATP) and thus number of metabolically active cells post nanoparticle exposure; the LDH assay measures the amount of lactate dehydrogenase released by damaged cells into the media. Experimental controls were defined as plain cells in media and pure media with and without nanoparticles. Toxicity assessment and calculations were conducted according to the manufacturers' protocols. Statistical analysis was performed using JMP analysis of variance (ANOVA) to compare results between groups. Tukey's post hoc test was used in conjunction with ANOVA for further analysis. Differences were recorded to be statistically significant at $p<0.05$. All errors are given as standard deviations.

\section{Results and Discussion}

\section{Structure, Formation, and Elemental Composition of} Icosahedra GGS NP

Original models based upon the Mie theory have suggested that the NIR SPR associated with GGS NP are due to a hexagonal core-shell structure [10]. More recent reports have hypothesized that the NIR peak should actually be attributed to gold nanoparticle aggregates, thus suggesting that the GGS NP are, therefore, not comprised of a core-shell configuration [12,13]. To further elucidate the formation of GGS NP, we first used conventional formulations with microwave irradiation, halting the reaction (via quenching) immediately after nucleation was initiated. Microwave irradiation has been used extensively by many researchers to accelerate nucleation [29]. The quenched reaction formed a monodisperse population of small gold colloid, as evidenced by the single absorption peak at $520 \mathrm{~nm}$ (Fig. 1). By comparison, when the same microwave-assisted reaction was allowed to proceed for $1 \mathrm{~h}$ at room temperature, the same peak at $520 \mathrm{~nm}$ remains and a second, red-shifted $(\sim 620 \mathrm{~nm})$ peak becomes evident. Previously, it was stated that this second peak was due to the formation of the gold-coated gold sulfide nanoparticles [10]. However, high-resolution TEM (HR-TEM) images of the resulting particles show homogeneous icosahedral nanostructures with defined facets (Fig. 2). This conclusion is further confirmed by the tilting of the TEM stage; as the angle changes, different facets 


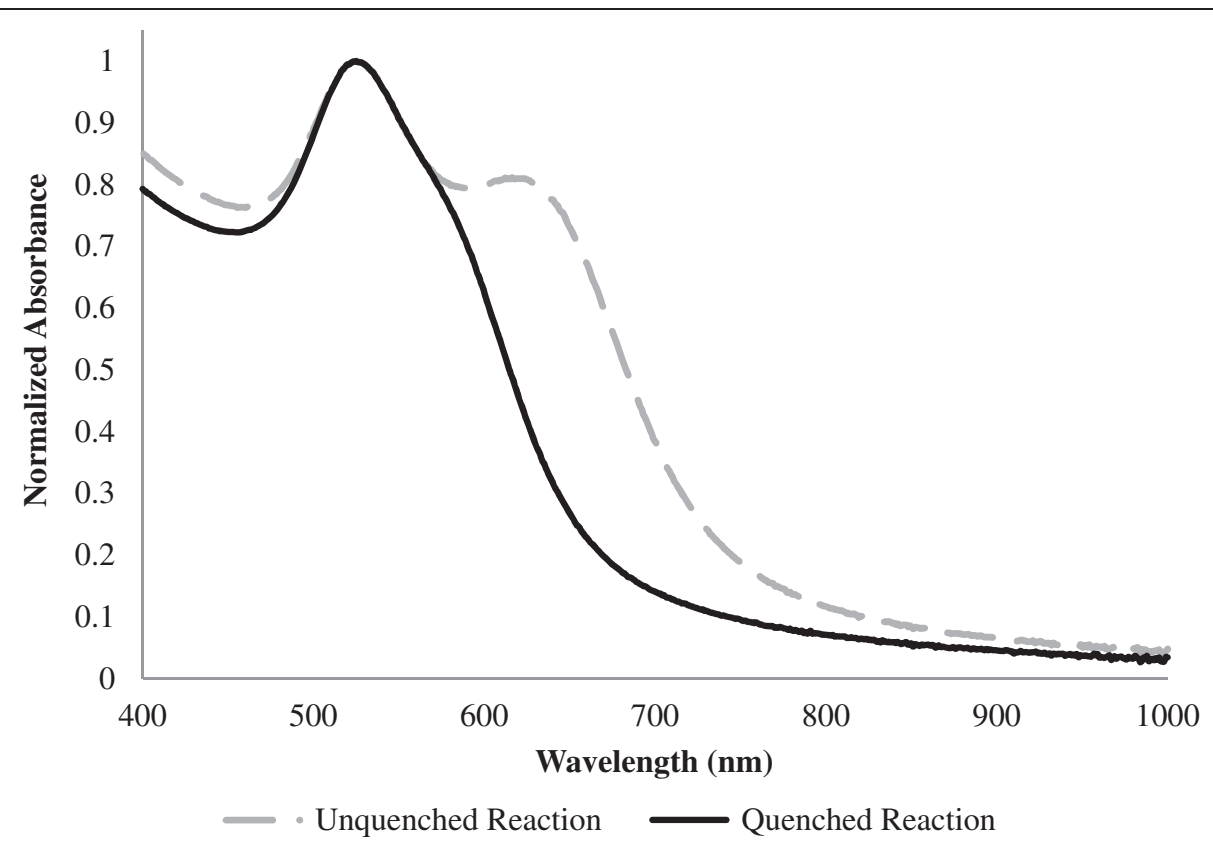

Fig. 1 Microwave-assisted nanoparticle synthesis. The quenched reaction resulted in the formation of only gold nuclei (520-nm peak) indicative of small gold colloid. The unquenched reaction produced an additional peak $(620 \mathrm{~nm})$ originally thought to be associated with the formation of gold-coated gold sulfide nanoparticles

become focused and defined, thus confirming a 3D icosahedra structure with no evidence of a core-shell configuration [30]. Furthermore, the second absorption peak at $620 \mathrm{~nm}$ is not consistent with reported spectra for gold-coated gold-sulfide nanocolloids, found to be near $790 \mathrm{~nm}$ [4]. These results collectively suggest that no gold sulfide nanoparticles are being produced at this stage but rather the icosahedral species of the conventional one-pot method.
We next used standard GGS NP fabrication procedures and determined the elemental composition of both icosahedra particles and nanoplates using EDS, FFT, and SAED analysis ( $n=4$ particles per analysis). These two particle types were analyzed since the standard GGS NP fabrication procedure results mostly in both particle populations. Based on this analysis, the nanoplates and icosahedra particles produced identical spectra consistent with face center cubic (FCC) gold (Fig. 3). The largest peaks are

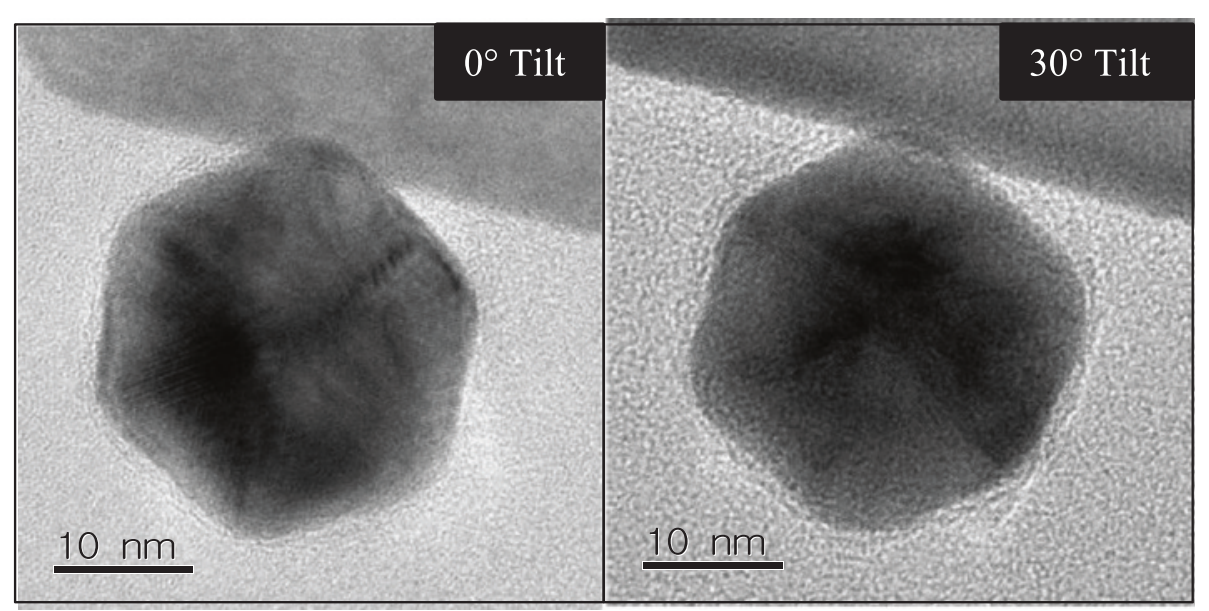

Fig. 2 TEM of icosahedral nanoparticles with $30^{\circ}$ tomography tilt angle. With the stage tilt, different facets become visible, in addition to the change in crystal orientation, confirming the 3D structure of the icosahedral nanoparticles. Scale bars represent $10 \mathrm{~nm}$ 


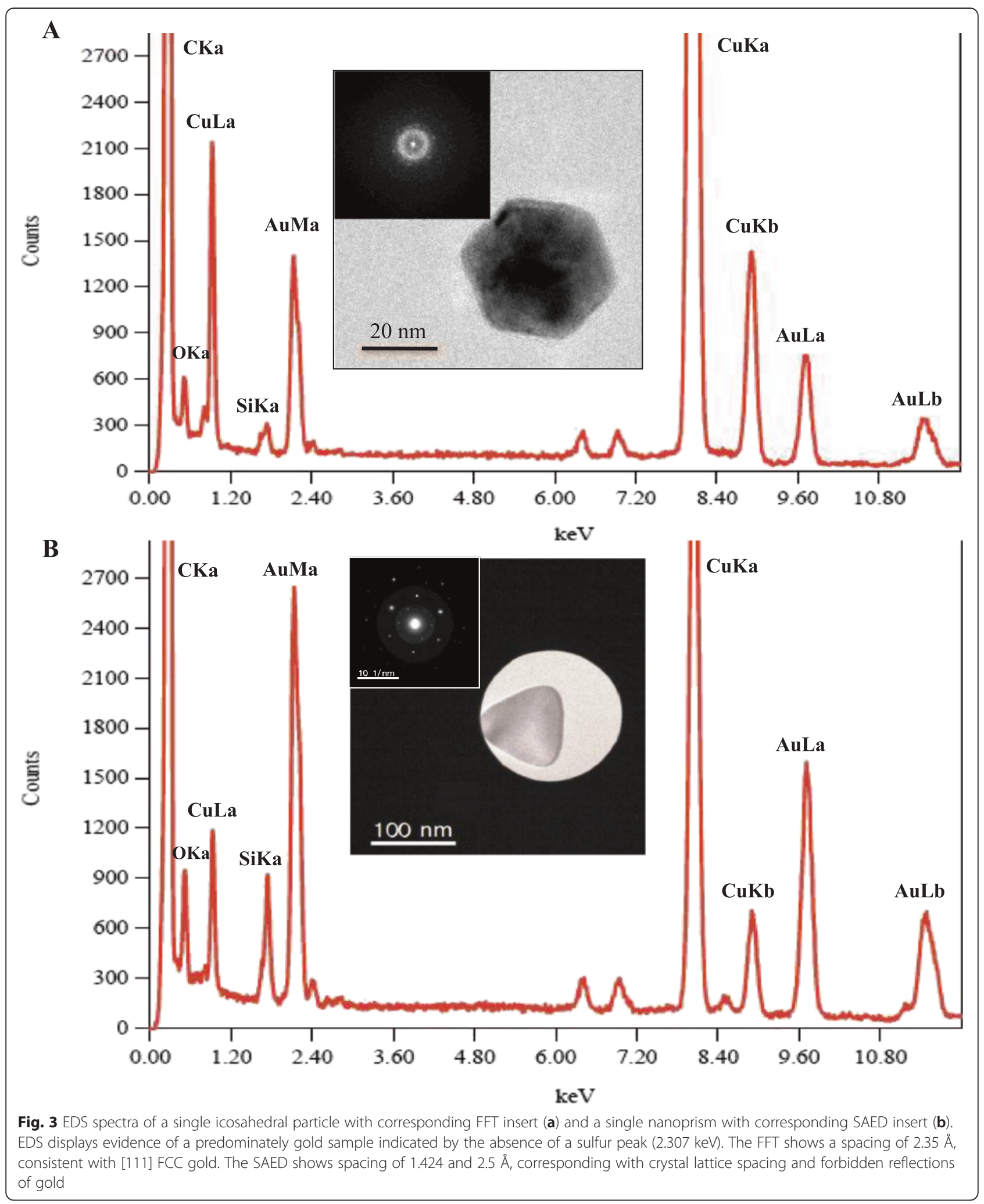

carbon $(\mathrm{C})$ and copper $(\mathrm{Cu})$, components of the TEM grid, and iron (Fe) and cobalt (Co), which are constituents of the TEM grid holder. Outside of the extraneous peaks, the peaks of interest are all derivatives of gold with no evidence of sulfur, which has a characteristic EDS peak at $2.307 \mathrm{keV}$. If sulfur was present in the sample, it is present 
at less than one part per billion, providing no significant contribution to the particle structure nor role in its bulk material properties. SAED and FFT analyses showed the predominant lattice spacing correspond to FCC [111] and [100] $\mathrm{Au}$, with some forbidden reflections that can be indexed as $1 / 2$ [422] FCC Au [31]. Forbidden reflections are common in highly faceted gold nanoparticles with an artifact that results in a lattice spacing of $2.5 \AA$. These characterization results indicate that both nanoparticle populations (icosahedra and nanoplates) are predominately gold with negligible amounts of sulfur. These results support previous claims that sodium thiosulfate acts as both a reducing agent and a shape-directing agent, facilitating growth of nanoparticles predominately into their preferred highly faceted multiple-twinned structure with mainly [111] and [100] facets [32]. Formation of nanoplates is most likely due to insufficient amounts of sodium thiosulfate as well as nucleation and growth occurring simultaneously.

\section{Seed-Mediated GGS NP Synthesis}

To improve the monodispersity of GGS NP, we created GGS NP based on seed-mediated growth, as this method allows for greater synthesis control. Seed-mediated growth is often used to increase control over the reaction conditions due to the separation of nucleation and growth steps [33]. Small gold seeds $(5 \mathrm{~nm})$ were successfully synthesized as previously reported [24]. The optimal seed-mediated condition involved incubation of sulfide with 5-nm gold colloid for $24 \mathrm{~h}$ and then adding additional gold salt. Colloid that was not aged resulted in globular, ill-defined particles, indicating the importance of sulfide in determining the overall shape of the particles (data not shown). The seed-mediated method resulted in a NIR peak located at $717 \mathrm{~nm}$ (Fig. 4), which was much lower and blue-shifted compared to the conventional synthesis which results in a NIR peak $\sim 800 \mathrm{~nm}$. Corresponding TEM images show that the distribution of the nanoplates resulting from seed-mediated synthesis was significantly reduced (by $\sim 50 \%$ ) compared to the conventional one-step synthesis method. As shown in Fig. 4, the seed-mediated sample consisted predominately of icosahedral nanoparticles, which made up approximately $82 \%$ of the entire sample. While nanoplates were still formed using this seed-mediated method, these particles were smaller $(\sim 50 \mathrm{~nm})$ and fewer in number than that produced by the conventional method. This provided the first indication that the icosahedral nanoparticles were not the species contributing to the NIR peak, which is seen with the conventional one-step synthesis method. While the seed-mediated method resulted in a minor NIR peak $(\sim 717 \mathrm{~nm})$, TEM results suggest that the 717-nm peak may be due to the inclusion of smaller and less concentrated nanoplates. In a conventional reaction, the NIR peak is a result of the aggregation between icosahedral and nanoplate particles, producing one combined peak near $800 \mathrm{~nm}$ $[11,12]$. These results indicate that the corresponding

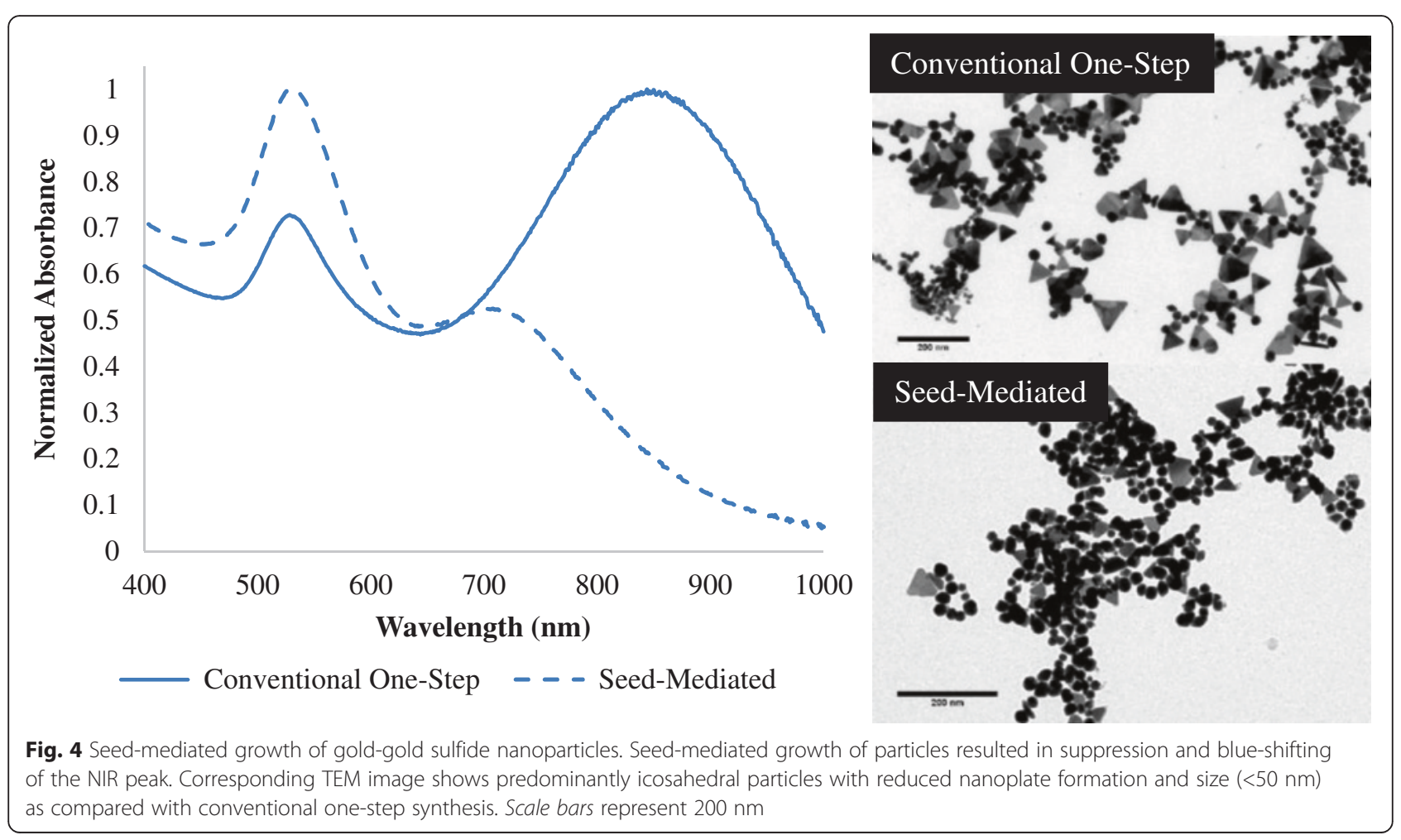


proportion of icosahedral to nanoplate phenotypes is responsible for the blue- or red-shifting of the NIR peak.

\section{Elucidation of Temperature Effects on NIR-Contributing GGS NP Species}

We further examined the effects of temperature alterations to control conventional reaction kinetics to confirm the NIR-contributing species. The effect of varying fabrication temperature (from 0 to $50{ }^{\circ} \mathrm{C}$ ) on the SPR is shown in Fig. 5. Traditional fabrication of GGS NP occurs at room temperature $\left(25^{\circ} \mathrm{C}\right)$. This synthesis condition results in an optimal SPR peak for photothermal therapy $(\sim 800 \mathrm{~nm})$ yet comprises significant shape variations, consistent with previously published results [5]. At a higher fabrication temperature of $50{ }^{\circ} \mathrm{C}$, the traditional NIR peak is replaced by a reduced peak at $\sim 650 \mathrm{~nm}$ with a dominant 520-nm peak, suggesting that higher temperatures yield less of the NIR-contributing species. At high temperatures, the gold precursor experiences an increased nucleation rate, increasing the reaction kinetics and reduction rate [34-36]. Thus, the formed particles are likely to be smaller and icosahedral in shape, due to a reduced growth rate as a result of the increased reduction [34]. Conversely, for considerably lower temperatures $\left(0{ }^{\circ} \mathrm{C}\right)$, the NIR peak red shifts and broadens beyond the $900-\mathrm{nm}$ range, with the absorbance peak at $520 \mathrm{~nm}$ decreasing in intensity. This data is in agreement with results presented by James et al. [22] which showed that wavelength increased (red-shifted) with decreasing temperatures to tune the peak absorbance of gold nanoplates beyond $900 \mathrm{~nm}$. The larger particles (gold nanoplates) may be formed as a result of the decreased rate of reduction at these lower temperatures. TEM images reveal the predominance and increased size of nanoplates synthesized at $0{ }^{\circ} \mathrm{C}$ compared to a dramatic increase in icosahedral nanoparticle formation at $50{ }^{\circ} \mathrm{C}$. While nanoplates are still formed at $50{ }^{\circ} \mathrm{C}$, the predominance of icosahedral nanoparticles likely results in the suppression of the characteristic gold nanoplate NIR plasmon resonance observed at room temperature. This study suggests that the presence of the nanoplates contributes substantially to the NIR SPR wavelength and intensity using the conventional one-step GGS NP synthesis.

\section{Size-Selective Separation of Polydisperse Samples}

To support our hypothesis that the nanoplates are the only particles contributing to the NIR peak, we used microfiltration to isolate different nanoparticle populations. Previous studies have traditionally purified NIRgold nanoparticle mixtures through multiple centrifugation steps and more recently utilized regenerated cellulose membranes (RCM) in a novel synthesis process named "DiaSynth" [22, 37]. In these studies, we wanted to create a homogenous nanoparticle suspension by using a rapid filtration method following a conventional one-pot synthesis, for the confirmation of the NIR-contributing species. To isolate the different nanoparticle subtypes, we employed both glycerol density separation and sequential micron pore filtration. Glycerol density separation has been widely published in the literature and has been used for many different nanoparticle applications, as it allows the separation of bare particles [25]. However, residual glycerol must be removed after separation, requiring multiple wash cycles. For the GGS NP, the optimal conditions

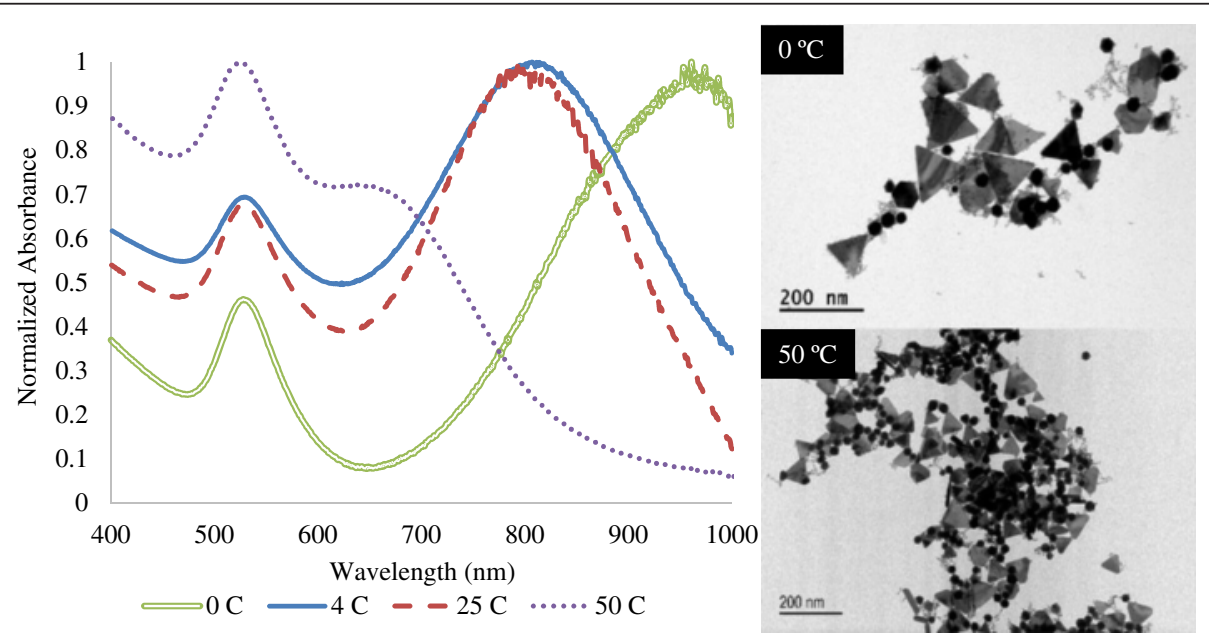

Fig. 5 Effect of temperature on conventional one-step gold-gold sulfide synthesis. Room temperature reaction provides characteristic NIR peak $(800 \mathrm{~nm})$ and minimal gold colloid peak $(520 \mathrm{~nm})$ with the production of polydisperse nanoparticles. At lower temperatures $\left(0^{\circ} \mathrm{C}\right)$, the presence of larger gold nanoplates results in red-shifting and broadening of the NIR peak. At higher temperatures $\left(50^{\circ} \mathrm{C}\right)$, the dramatic increase in icosahedral nanoparticles results in diminished NIR peak. Scale bars represent $200 \mathrm{~nm}$ 
for particle separation in glycerol were $40 \mathrm{~min}$ at $3200 \mathrm{~g}$ and $40{ }^{\circ} \mathrm{C}$ using a $30-90 \%$ concentration gradient. This resulted in clean separation bands with minimal streaking, as shown in Fig. 6. Samples were carefully handled in order to remove the desired nanoparticle subsets and avoid the blending of bands. This process was difficult to reproduce, as any disruption in sample preparation would inappropriately mix the bands. In this regard, extraction of the bands was prone to manual error, increasing the likelihood of cross-over between separated samples. Density filtration demonstrated that the largest particles were predominately nanoplates, whose corresponding spectra revealed a NIR peak near $795 \mathrm{~nm}$. Equally, small density bands consisted primarily of icosahedrashaped nanoparticles, which were previously suggested as the NIR-contributing species, whose plasmon peak was approximately $680 \mathrm{~nm}$. While nanoplates were present in fraction A, as previously suggested, the predominance of the icosahedral nanoparticles likely results in a relative reduced plasmon peak.

In addition to glycerol density separation, we performed sequential micron pore filtration. This technique was used to filter both bare and PEGylated particles, both of which passed efficiently through the filters (Fig. 7).

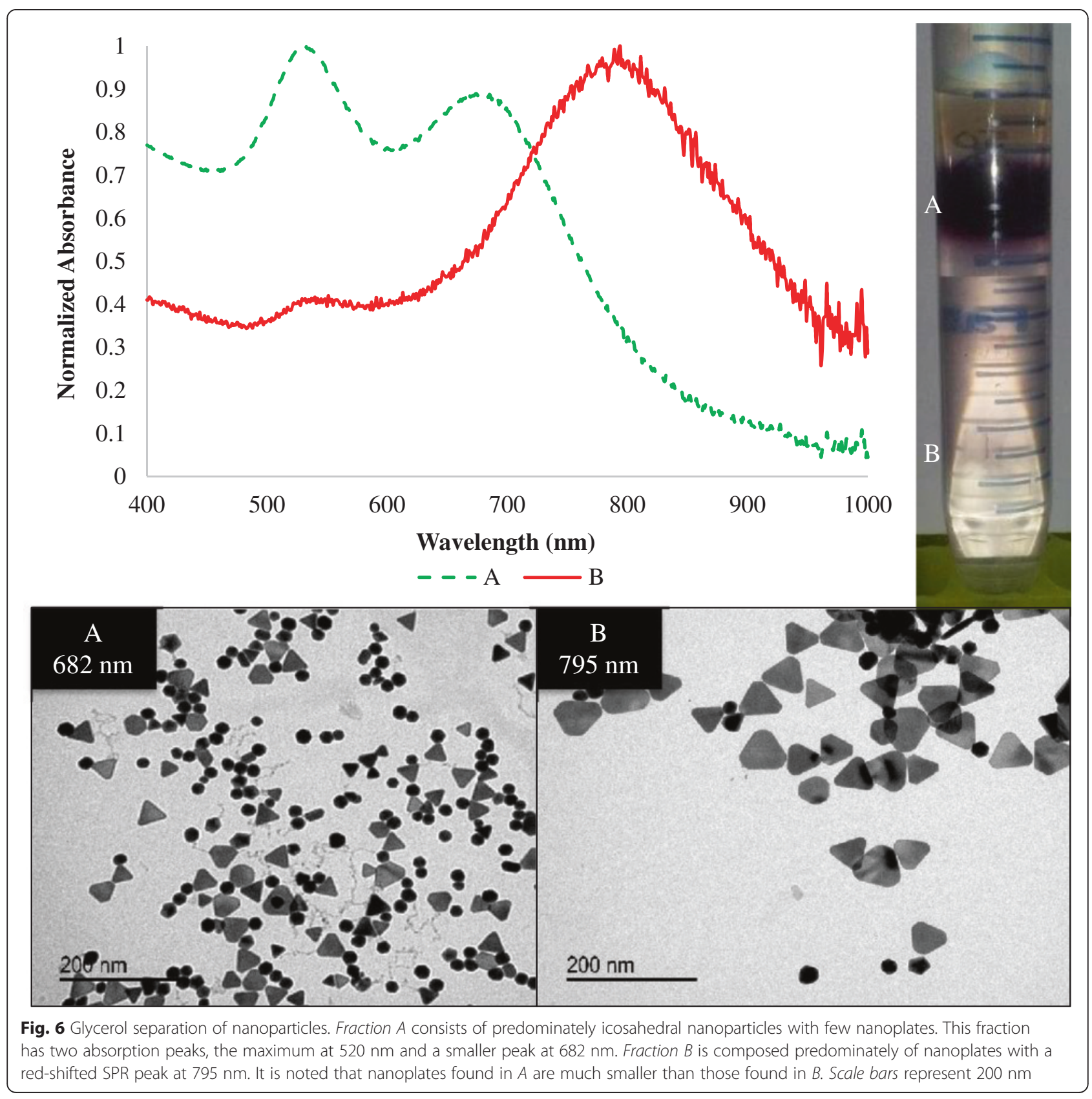




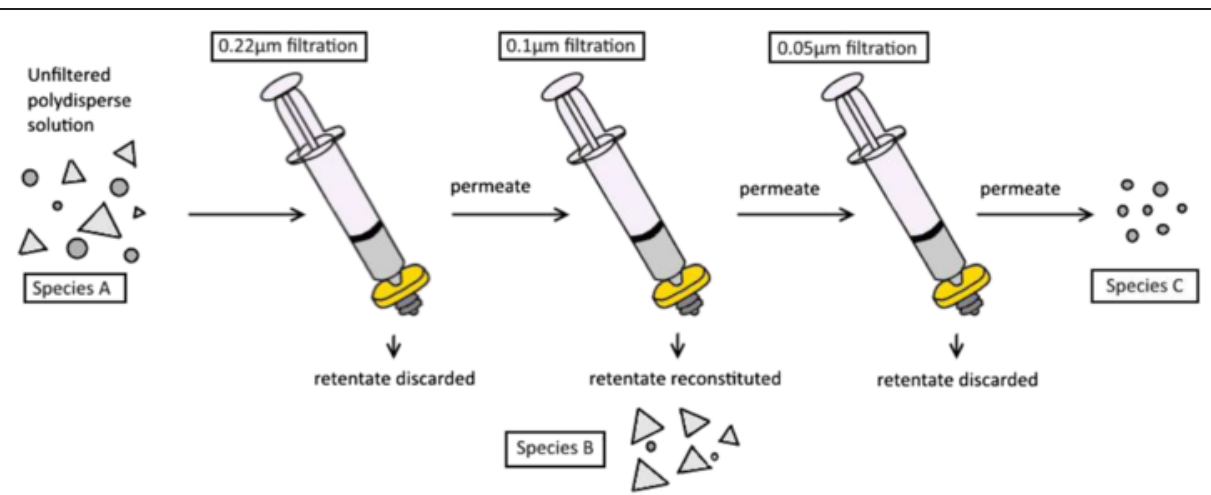

Fig. 7 Microfiltration process of polydisperse samples. Samples of interest, species $B$ and $C$ were collected via sequential filtration steps. Species $B$ includes all particles retained by the $0.1-\mu \mathrm{m}$ filter. Species $C$ contains particles which passed through the $0.05-\mu \mathrm{m}$ filter

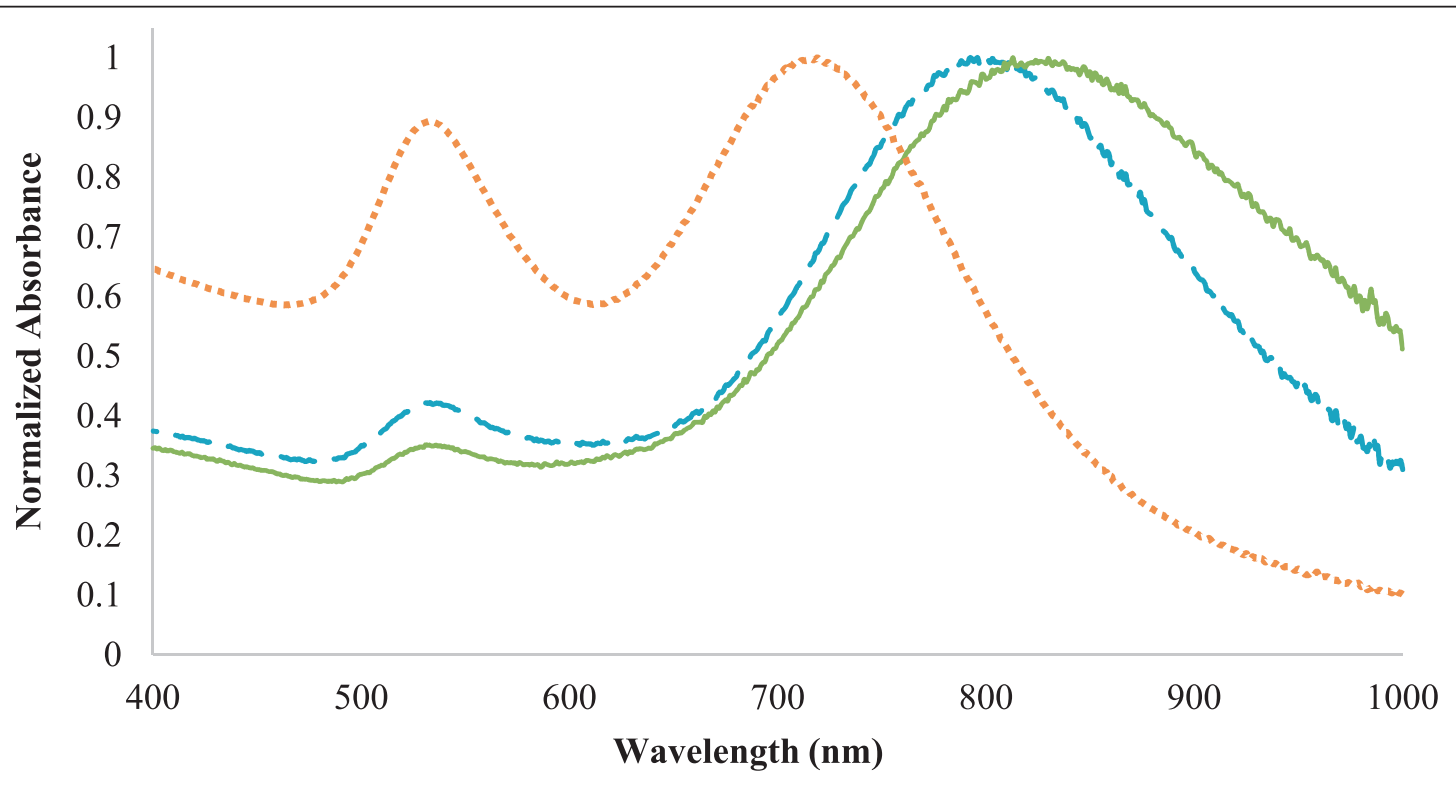

- - Species A $\longrightarrow$ Species B $\quad \cdots \cdots$ Species C

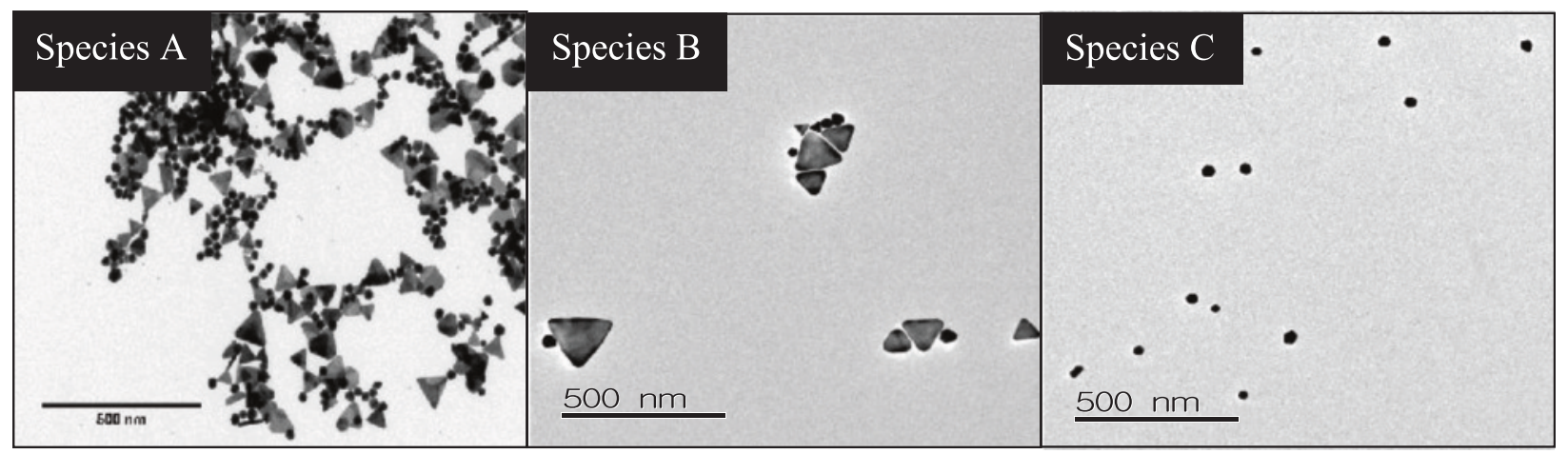

Fig. 8 Selective size filtration of polydisperse nanoparticles. Sequential filtering results in blue-shifting of the NIR peak and removal of the larger nanoplates. Scale bars shown represent $500 \mathrm{~nm}$ 
Table 1 Nanoparticle size, NIR peak, and nanoplate composition

\begin{tabular}{llll}
\hline Sample & $\begin{array}{l}\text { Diameter } \\
\text { size }(\mathrm{nm})\end{array}$ & NIR peak $\lambda(\mathrm{nm})$ & $\begin{array}{l}\text { Nanoplate } \\
\text { composition (\%) }\end{array}$ \\
\hline Species A & $10-300$ & 800 & 26 \\
Species B & $100-220$ & 834 & 57 \\
Species C & $<50$ & 705 & 23 \\
\hline
\end{tabular}

To recover the retained nanoparticles, the filters were sonicated in a water bath to release captured particles. This simple technique resulted in the quickest, most efficient separation of nanoparticles compared to other methods. The two filtered samples of interest were those recovered from the $0.1-\mu \mathrm{m}$ filter and those that passed through the $0.05-\mu \mathrm{m}$ filter, species B and C, respectively. These two filtered species were compared to the polydisperse sample produced via the conventional one-pot synthesis, species A. As shown in Fig. 8, species $C$ has a SPR peak at $705 \mathrm{~nm}$ resembling that of a primarily icosahedral particle sample theorized from Fig. 1. Species B is red-shifted with a NIR SPR peak at
$834 \mathrm{~nm}$. TEM images analyzed through Image demonstrated that species $\mathrm{C}$, the most blue-shifted fraction, consisted predominately of icosahedral particles ( $\sim 7 \%$ ) while species $B$, the most red-shifted fraction consisted largely of nanoplates ( 57\%). These results, detailed in Table 1, support the hypothesis that nanoplates are the predominant species contributing to the NIR peak. This is supported by Millstone et al. who have identified nanoprisms as typically having SPRs throughout the visible and NIR regions by means of controlling prism thickness, edge length, and vertex sharpness [2]. While we primarily investigate the influence of nanoparticle composition on the resulting SPR, Millstone et al. draw attention to the blue- or redshifting of nanoplate SPRs as a result of adjusting structural variables (edge length, thickness, and degree of truncation) [2]. The size separation of the polydisperse nanoparticle samples and examination of corresponding UV-vis spectra in tandem with TEM images confirms the contribution of the nanoplates to the NIR SPR, not the icosahedra nanoparticles.

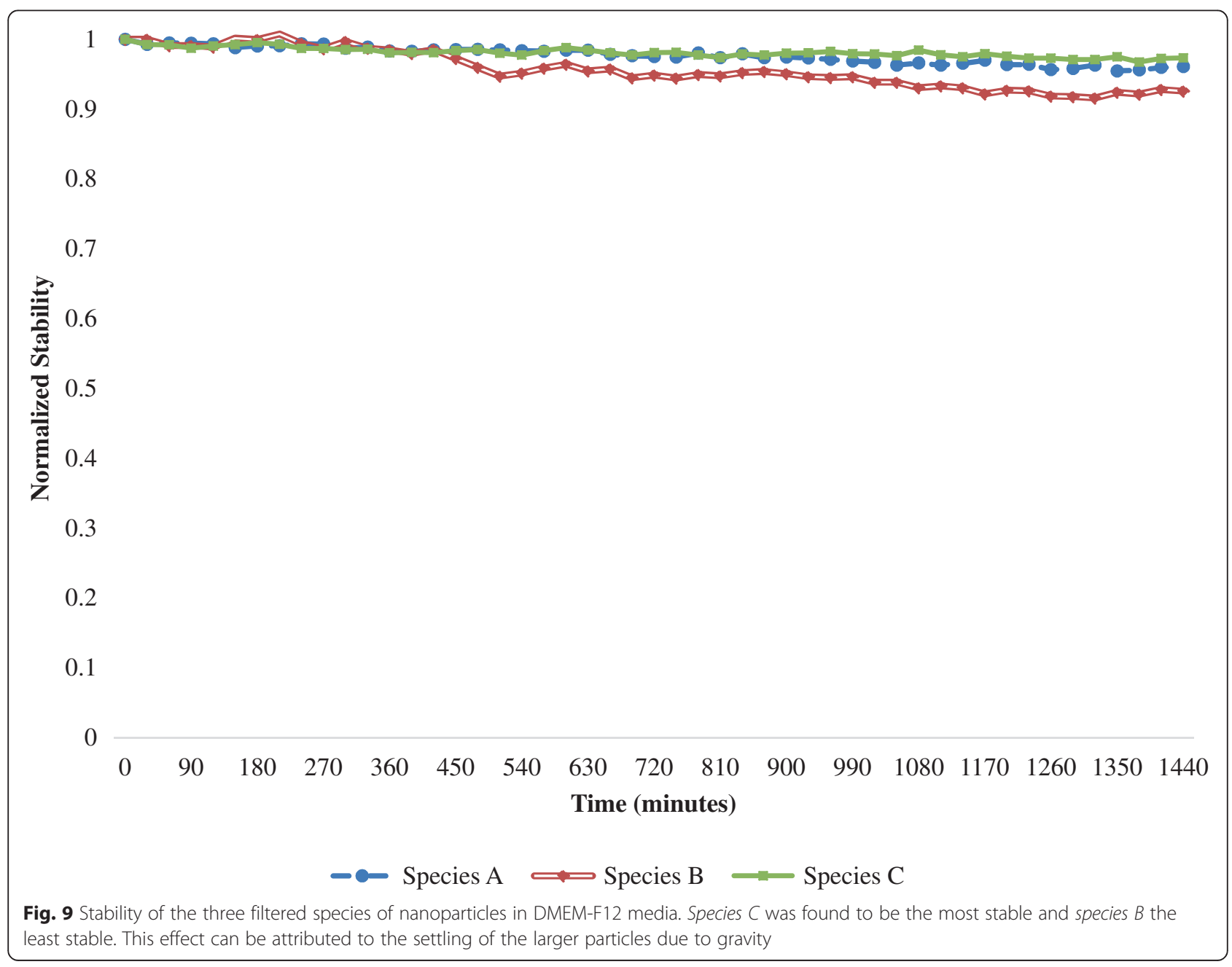




\section{GGS NP Toxicity Studies}

Nanoparticle size effects are well known to greatly affect cellular toxicity as well as corresponding biodistribution profiles [38]. Studies examining the effect of gold nanoparticle size on biodistribution have shown that small particles $(10 \mathrm{~nm})$ predominately distribute and localize in the blood, liver, kidney, heart, and brain, whereas larger particles $(50-250 \mathrm{~nm})$ were only detected in the blood, liver, and spleen [39]. These size effects are confounded by additional shape variations, which have different charges, defects, and surface areas compared to uniform samples, resulting in unpredictable behaviors in vivo [40]. Bismuth selenide $\left(\mathrm{Bi}_{2} \mathrm{Se}_{3}\right)$ nanoplates, $60 \mathrm{~nm}$ in diameter, were previously shown to accumulate in the liver, spleen, and kidney [41]. Previous studies have also demonstrated the preferential uptake in

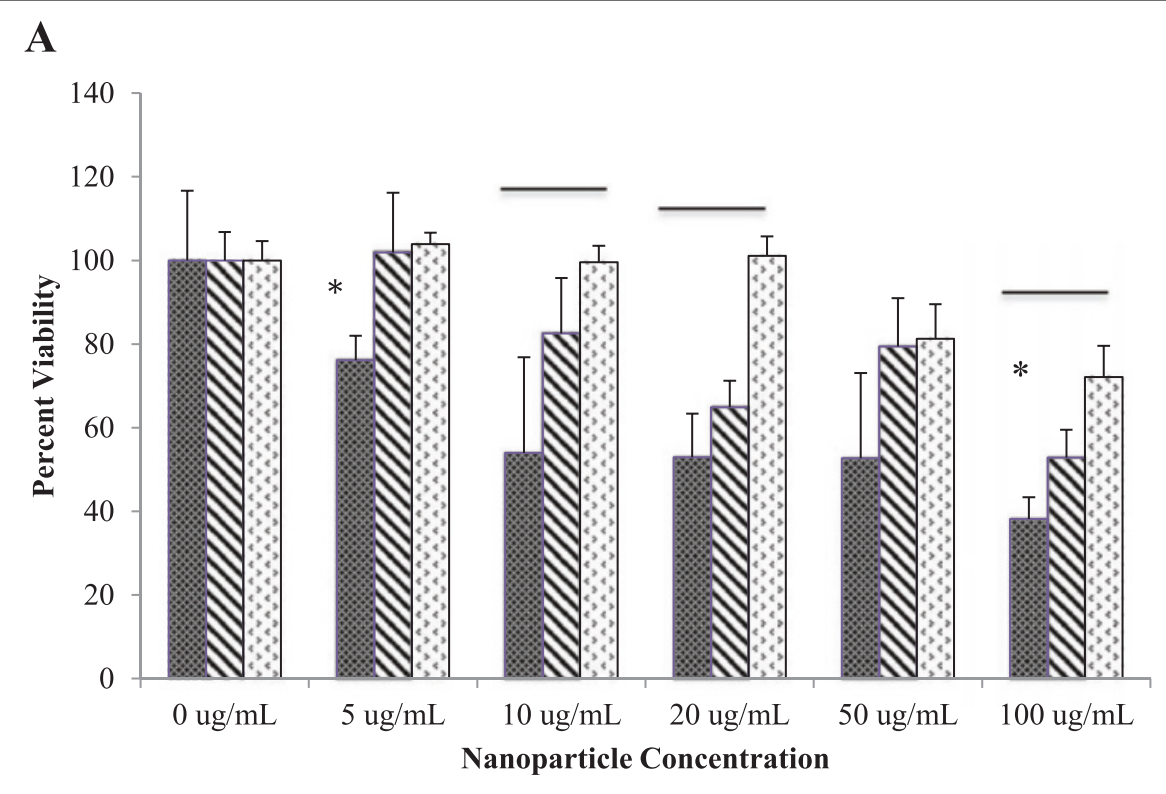

B

Species A $\mathbf{\Delta}$ Species B $\square$ Species C

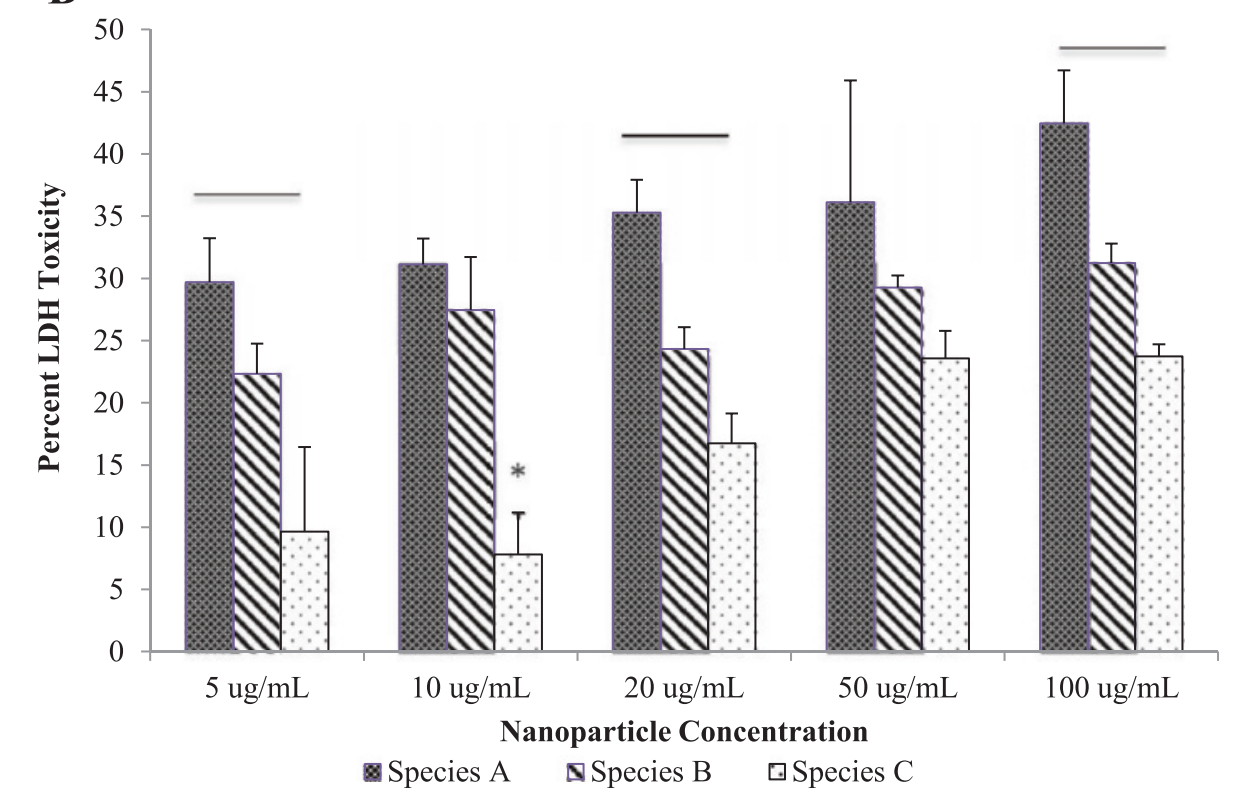

Fig. 10 Cytotoxicity of nanoparticles to RPE-1 cells: Celltiter-glo (a) and LDH assay (b). The non-filtered, polydisperse particles displayed the most cytotoxicity while the more homogeneous and smaller particles were shown to be the least toxic. Line above samples indicates that all three values are statistically significant $(p<0.05)$. Asterisk denotes one sample which is statistically different from the other two $(p<0.05)$. Error bars represent standard deviation 
macrophages of spherical particles over those with high aspect ratios [42].

To determine the impact of sample purification on cytotoxicity, the aforementioned three different nanoparticle populations (species A, B, and C) were evaluated. Samples were prepared at comparable concentrations ranging from 5 to $100 \mu \mathrm{g} / \mathrm{mL}$. Prior to conducting experiments, particle stability in culture media was analyzed. Toxicity effects can be skewed by the effect of nanoparticles settling in the media over time. While all samples were stable for $24 \mathrm{~h}$, the nanoplate fraction (species B) showed the least stability, as evidenced by the greater temporal decrease in the maximum peak absorbance (Fig. 9). This effect is expected due to the larger size, and increased surface area, of the nanoplates, resulting in increased settling and possible protein adsorption. However, this effect only resulted in $6 \%$ loss in stability. Using both cell viability and LDH assays, our analyses showed that species A resulted in the greatest cytotoxicity, whereas species $C$ resulted in the least toxicity (Fig. 10). While previous studies have stated that smaller particles are more toxic than larger nanoparticles of the same shape due to enhanced surface area, shape effects tend to dominate [38, 34]. Specifically, nanoplates are reportedly more toxic than spherical particles due to their propensity for surface defects [40]. However, our results suggest that polydispersity (with the most variation in both size and shape) may result in the greatest cytotoxicity. While we demonstrate that variations in both shape and size impact toxicity using simplified 2D assays, these effects are also likely to be exacerbated when moving into 3D assays and animal models. Sample polydispersity not only affects cellular uptake and toxicity but likely provides undesirable variations in biodistribution in vivo [42]. These findings further dictate the need to produce well characterized and monodisperse samples prior to advancing to clinical nanomedicine trials.

\section{Conclusions}

Conventional one-step synthesis of NIR GGS NP was originally proposed as having an icosahedral, sulfide coregold shell nanostructure, yet fabrication results in a polydisperse solution of nanoplates, icosahedral nanoparticles, and irregularly shaped asymmetric nanoparticles. Subsequent size-selective separation and compositional analysis reveal that the elemental composition of these particles is FCC gold and that nanoplates are the predominant NIRcontributing species, not the icosahedral nanoparticles as originally hypothesized. Here, we optimized two filtration methods, both of which facilitated nanoparticle separation, with microfiltration providing the most reproducible and rapid fractionation. Importantly, the original polydisperse samples produced by the conventional onestep reduction of chloroauric acid by sodium thiosulfate resulted in enhanced cellular toxicity in vitro compared to more purified samples. While further work is needed to elucidate the toxic properties of nanomaterials universally, this study highlights the need to fully characterize and optimize nanomaterials before translating into more complex and biologically relevant animal and pre-clinical models. This upstream analysis will expedite the development of highly promising translational nanomaterials for in vivo applications and ultimate FDA approval.

\section{Abbreviations}

NIR: near-infrared; TEM: transmission electron microscopy; EDS: energy dispersive x-ray spectroscopy; FCC: face center cubic; SPR: surface plasmon resonance; GGS NP: gold-gold sulfide nanoparticle; FDA: Food and Drug Administration; OD: optical density; SAED: selected area electron diffraction; FFT: fast Fourier transform; RPE-1: hTERT-RPE-1; LDH: lactose dehydrogenase; ATP: adenosine triphosphate; ANOVA: analysis of variance.

\section{Competing Interests}

The authors declare that they have no competing interests.

\section{Authors' Contributions}

SS and LMR carried out the synthesis of gold nanoparticles and optical measurements. SS completed the stability studies. LMR completed size-selective separation and toxicity studies. SS, LMR, PJK, and LRB prepared the manuscript. LRB supervised the experimental design, data interpretation, and discussions. PJK provided assistance with the experimental design. DC provided the RPE-1 cells and participated in the interpretation of corresponding experimental data and discussions as well as editing of the manuscript. All authors read and approved the final manuscript.

\section{Authors' Information}

SS is a bachelor degree student from the Department of Biological Systems Engineering, Virginia Polytechnic and State Institute. LMR and LRB are from the Department of Biomedical Engineering and Mechanics, Virginia

Polytechnic and State Institute. LMR has graduated with a master's degree. LRB is an assistant professor. LMR, PJK, and LRB are affiliates of the Virginia Tech Center for Sunstainable Nanotechnology. PJK is from the Department of Civil and Environmental Engineering, Virginia Polytechnic and State Institute. PJK is a professor. DC is from the Department of Biological Sciences and Virginia Bioinformatics Institute, Virginia Polytechnic and State Institute. DC is an associate professor.

\section{Acknowledgements}

The authors would like to thank Chris Winkler from the Nanoparticle Characterization and Fabrication Laboratory (NCFL) for the assistance with nanoparticle characterization and imaging. We also thank Kathy Lowe (Virginia Maryland College of Veterinary Medicine) for the assistance with TEM imaging and Krystal Le (Department of Biochemistry) for the help with nanoparticle fabrication. Special thanks to the Virginia Tech Institute for Critical Technology and Applied Science (ICTAS) and the Virginia Tech Center for Sustainable Nanotechnology for the generous funding support.

\section{Author details}

'Department of Biological Systems Engineering, Virginia Tech, Seitz Hall, Blacksburg, VA 24061, USA. Department of Biomedical Engineering and Mechanics, Virginia Tech, 325 Stanger Street, 310 Kelly Hall (MC 0298), Blacksburg, VA 24061, USA. ${ }^{3}$ Virginia Tech Center for Sustainable Nanotechnology, Virginia Tech, Kelly Hall, Blacksburg, VA 24061, USA. ${ }^{4}$ Department of Biological Sciences and Virginia Bioinformatics Institute, Virginia Tech, 1015 Life Science Circle, Blacksburg, VA 24061, USA. ${ }^{5}$ Department of Civil and Environmental Engineering, Virginia Tech, Durham Hall, Blacksburg, VA 24061, USA. 'Department of Mechanical Engineering, Virginia Tech, Kelly Hall, Blacksburg, VA 24061, USA.

Received: 6 April 2015 Accepted: 15 May 2015

Published online: 28 May 2015 


\section{References}

1. Young JK, Figueroa ER, Drezek RA. Tunable nanostructures as photothermal theranostic agents. Ann Biomed Eng. 2012;40(2):438-59. doi:10.1007/s10439-011-0472-5.

2. Millstone JE, Hurst SJ, Metraux GS, Cutler JI, Mirkin CA. Colloidal gold and silver triangular nanoprisms. Small. 2009;5(6):646-64. doi:10.1002/smll.200801480

3. Kennedy LC, Bickford LR, Lewinski NA, Coughlin AJ, Hu Y, Day ES, et al. A new era for cancer treatment: gold-nanoparticle-mediated thermal therapies. Small. 2011;7(2):169-83. doi:10.1002/smll.201000134.

4. Ren $L$, Chow GM. Synthesis of nir-sensitive $A u-A_{2} S$ nanocolloids for drug delivery. Mater Sci Eng C. 2003;23(1):113-6. doi:10.1016/S0928-4931(02)00247-3.

5. Gobin AM, Watkins EM, Quevedo E, Colvin VL, West JL. Near-infraredresonant gold/gold sulfide nanoparticles as a photothermal cancer therapeutic agent. Small. 2010;6(6):745-52. doi:10.1002/smll.200901557.

6. Sun X, Zhang G, Patel D, Stephens D, Gobin AM. Targeted cancer therapy by immunoconjugated gold-gold sulfide nanoparticles using protein $\mathrm{G}$ as a cofactor. Ann Biomed Eng. 2012;40(10):2131-9. doi:10.1007/s10439-012-0575-7.

7. Day ES, Bickford LR, Slater JH, Riggall NS, Drezek RA, West JL. Antibodyconjugated gold-gold sulfide nanoparticles as multifunctional agents for imaging and therapy of breast cancer. Int J Nanomedicine. 2010;5:445-54.

8. Li Y, Gobin AM, Dryden GW, Kang X, Xiao D, Li SP, et al. Infrared light-absorbing gold/gold sulfide nanoparticles induce cell death in esophageal adenocarcinoma. Int J Nanomedicine. 2013;8:2153-61. doi:10.2147/ijn.s37140.

9. Zhou HS, Honma II, Komiyama H, Haus JW. Controlled synthesis and quantum-size effect in gold-coated nanoparticles. Phys Rev B Condens Matter. 1994;50(16):12052-6.

10. Averitt RD, Sarkar D, Halas NJ. Plasmon resonance shifts of Au-coated $A_{2} S$ nanoshells: insight into multicomponent nanoparticle growth. Phys Rev Lett. 1997;78(22):4217-20.

11. Zhang G, Jasinski JB, Howell JL, Patel D, Stephens DP, Gobin AM. Tunability and stability of gold nanoparticles obtained from chloroauric acid and sodium thiosulfate reaction. Nanoscale Res Lett. 2012;7(1):337. doi:10.1186/1556-276x-7-337.

12. Schwartzberg AM, Grant CD, van Buuren T, Zhang JZ. Reduction of HAuCl4 by $\mathrm{Na} 2 \mathrm{~S}$ revisited: the case for Au nanoparticle aggregates and against Au2S/Au core/shell particlest. J Phys Chem C. 2007:111(25):8892-901. doi:10.1021/jp067697g

13. Norman TJ, Grant CD, Magana D, Zhang JZ, Liu J, Cao D, et al. Near infrared optical absorption of gold nanoparticle aggregates. J Phys Chem B. 2002;106(28):7005-12. doi:10.1021/jp0204197.

14. Diao JJ, Chen $\mathrm{H}$. Near infrared surface plasmon resonance of gold tabular nanostructures in the HAuCl4-Na2S reaction. J Chem Phys. 2006;124(11):116103. doi:10.1063/1.2177661.

15. Likhatskii MN, Mikhlin YL. Influence of sulfide ions on the formation and properties of gold nanoparticles in aqueous solutions. Glas Phys Chem. 2007;33(4):422-5. doi:10.1134/S1087659607040189.

16. Morris T, Copeland H, Szulczewski G. Synthesis and characterization of gold sulfide nanoparticles. Langmuir. 2001;18(2):535-9. doi:10.1021/la011186y.

17. Clancy MK. Chapter 23-clinical translation and regulations of theranostics. In: Chen X, Wong S, editors. Cancer Theranostics. Oxford: Academic; 2014. p. 439-56.

18. Young J, Drezek R, editors. Synthesis of Au2S/Au Core/shell nanostructures Optics in the life sciences. Monterey, California: Optical Society of America: 2011. 2011/04/04.

19. Majimel J, Bacinello D, Durand E, Vallée F, Tréguer-Delapierre M. Synthesis of hybrid gold-gold sulfide colloidal particles. Langmuir. 2008;24(8):4289-94. doi:10.1021/la702829w.

20. Pelaz B, Grazu V, Ibarra A, Magen C, del Pino P, de la Fuente JM. Tailoring the synthesis and heating ability of gold nanoprisms for bioapplications. Langmuir. 2012;28(24):8965-70. doi:10.1021/la204712u.

21. Patel D. A novel high yield process for gold sulfide nanoparticle synthesis via shifting equilibrium of self-assembly reaction: University of Louisville. 2012.

22. James KT, OToole M, Patel D, Zhang G, Gobin AM, Keynton RS. A high yield, controllable process for producing tunable near infrared-absorbing gold nanoplates. RSC Advances. 2015. doi:10.1039/C4RA14889D.

23. Choi HS, Frangioni JV. Nanoparticles for biomedical imaging: fundamentals of clinical translation. Mol Imaging. 2010;9(6):291-310.
24. Haiss W, Thanh NT, Aveyard J, Fernig DG. Determination of size and concentration of gold nanoparticles from UV-vis spectra. Anal Chem. 2007;79(11):4215-21. doi:10.1021/ac0702084.

25. Wu W, Huang J, Wu L, Sun D, Lin L, Zhou Y, et al. Two-step size- and shape-separation of biosynthesized gold nanoparticles. Sep Purif Technol. 2013;106:117-22. http://www.sciencedirect.com/science/article/pii/ S1383586613000208

26. Hirsch LR, Halas NJ, West JL. Whole-blood immunoassay facilitated by gold nanoshell-conjugate antibodies. Methods Mol Biol. 2005;303:101-11. doi:10.1385/1-59259-901-x:101.

27. Laboratories C. Infinity telomerase-immortalized cell line culturing guide. 1999.

28. Gliga AR, Skoglund S, Wallinder IO, Fadeel B, Karlsson HL. Size-dependent cytotoxicity of silver nanoparticles in human lung cells: the role of cellular uptake, agglomeration and Ag release. Part Fibre Toxicol. 2014;11:11. doi:10.1186/1743-8977-11-11.

29. Van der Eycken EV. Aqueous microwave assisted chemistry. Synthesis and catalysis. RSC green chemistry series. In: Polshettiwar V, Varma RS, editors. Angew Chem Int Ed. 2010;49(52):10039-40. doi:10.1002/anie.201006427.

30. Chen HM, Liu RS, Jang LY, Lee JF, Hu SF. Characterization of core-shell type and alloy $\mathrm{Ag} / \mathrm{Au}$ bimetallic clusters by using extended $\mathrm{X}$-ray absorption fine structure spectroscopy. Chem Phys Lett. 2006;421(1-3):118-23. http://dx.doi.org/10.1016/j.cplett.2006.01.043.

31. Castaño V, Gómez A, Yacaman MJ. Microdiffraction and surface structure of small gold particles. Surf Sci Lett. 1984;146(2-3):L587-92. http://dx.doi.org/10.1016/0167-2584(84)90756-4.

32. Marks LD. Experimental studies of small particle structures. Rep Prog Phys. 1994;57(6):603.

33. Daeha S, Hyunjoon S. Synthesis of gold nanoparticles in liquid phase. Gold nanoparticles for physics, chemistry and biology. London: Imperial College Press; 2011. p. 103-38.

34. Kumar DVR, Kulkarni AA, Prasad BLV. Synthesis of triangular nanoplates: role of bromide ion and temperature. Colloids Surf A: Physiochem Eng Asp. 2013:422:181-90

35. Mountrichas G, Pispas S, Kamistos E. Effect on temperature on the direct synthesis of gold nanoparticles mediated by poly(dimethylaminoethyl methacrylate) homopolymer. J Phys Chem C. 2014;118:22754-9.

36. Liu H, Yang Q. A two-step temperature-raising process to gold nanoplates with optical and surface enhanced Raman spectrum properties. Cryst Eng Comm. 2011;13:2281.

37. Patel DN, James KT, OToole MG, Zhang G, Keynton RS, Gobin AM. A high yield, one-pot dialysis-based process for self-assembly of near infrared absorbing gold nanoparticles. J Colloids Interface Sci. 2015;441:10-6.

38. Pan Y, Neuss $S$, Leifert A, Fischler M, Wen F, Simon U, et al. Sizedependent cytotoxicity of gold nanoparticles. Small. 2007;3(11):1941-9. doi:10.1002/smll.200700378.

39. Krystek P. A review on approaches to bio distribution about gold and silver engineered nanoparticles by inductively coupled plasma mass spectrometry. Microchem J. 2012;105:39-43.

40. George S, Lin S, Ji Z, Thomas CR, Li L, Mecklenburg M, et al. Surface defects on plate-shaped silver nanoparticles contribute to its hazard potential in a fish gill cell line and zebrafish embryos. ACS Nano. 2012;6(5):3745-59. doi:10.1021/nn204671v

41. Zhang X-D, Chen J, Min Y, Park GB, Shen X, Song S-S, et al. Metabolizable Bi2Se3 nanoplates: biodistribution, toxicity, and uses for cancer radiation therapy and imaging. Adv Funct Mater. 2014;24(12):1718-29.

42. Toy R, Peiris PM, Ghaghada KB, Karathanasis E. Shaping cancer nanomedicine: the effect of particle shape on the in vivo journey of nanoparticles. Nanomedicine. 2014;9(1):121-34. 